\title{
Spin-orbit mediated control of spin qubits
}

\author{
Flindt, Christian; Sørensen, A.S; Flensberg, Karsten
}

Published in:

Physical Review Letters

Link to article, DOI:

10.1103/PhysRevLett.97.240501

Publication date:

2006

Document Version

Publisher's PDF, also known as Version of record

Link back to DTU Orbit

Citation (APA):

Flindt, C., Sørensen, A. S., \& Flensberg, K. (2006). Spin-orbit mediated control of spin qubits. Physical Review Letters, 97(24), 240501. https://doi.org/10.1103/PhysRevLett.97.240501

\section{General rights}

Copyright and moral rights for the publications made accessible in the public portal are retained by the authors and/or other copyright owners and it is a condition of accessing publications that users recognise and abide by the legal requirements associated with these rights.

- Users may download and print one copy of any publication from the public portal for the purpose of private study or research.

- You may not further distribute the material or use it for any profit-making activity or commercial gain

- You may freely distribute the URL identifying the publication in the public portal

If you believe that this document breaches copyright please contact us providing details, and we will remove access to the work immediately and investigate your claim 


\title{
Spin-Orbit Mediated Control of Spin Qubits
}

\author{
Christian Flindt, ${ }^{1,2}$ Anders S. Sørensen, ${ }^{1}$ and Karsten Flensberg ${ }^{1}$ \\ ${ }^{1}$ Niels Bohr Institute, Universitetsparken 5, DK-2100 Copenhagen, Denmark \\ ${ }^{2}$ MIC - Department of Micro and Nanotechnology, NanoDTU, Technical University of Denmark, \\ Building 345east, DK-2800 Kongens Lyngby, Denmark \\ (Received 21 March 2006; published 11 December 2006)
}

\begin{abstract}
We propose to use the spin-orbit interaction as a means to control electron spins in quantum dots, enabling both single-qubit and two-qubit operations. Very fast single-qubit operations may be achieved by temporarily displacing the electrons. For two-qubit operations the coupling mechanism is based on a combination of the spin-orbit coupling and the mutual long-ranged Coulomb interaction. Compared to existing schemes using the exchange coupling, the spin-orbit induced coupling is less sensitive to random electrical fluctuations in the electrodes defining the quantum dots.
\end{abstract}

PACS numbers: 03.67.Lx, 71.70.Ej, 73.21.La

It is believed that solid-state systems could facilitate large-scale quantum computing [1] due to the welldeveloped fabrication techniques that allow for a high degree of scalability. On the other hand, solid-state systems are inherently more noisy than, e.g., quantum optical systems, and, in particular, several sources of low-frequency noise are typically present in a solid-state environment. One prominent candidate for solid-state quantum computing uses electron spins in semiconductor quantum dots as carriers of the fundamental unit of information, the qubit [2]. Electron spins have the advantage that they are weakly coupled to the surroundings and therefore weakly sensitive to noise. At the same time, however, this weak coupling makes the electron spin hard to control experimentally. To couple two spin qubits, it was proposed to use the exchange coupling between electron spins in neighboring quantum dots [3], and this was recently demonstrated experimentally [4]. Here the triplet and singlet spin states have different charge profiles, thereby enabling electrical control of the coupling. Unfortunately, this spin-charge coupling also makes the qubits sensitive to electrical noise and, in particular, to low-frequency noise [5]. In this setting the spinorbit interaction is also considered as a source of decoherence [6], because it mixes spin and charge. Recently, however, it has been proposed that it could also play a role in the coherent interaction of qubits [7]. In this Letter we take these ideas further and propose to use the spinorbit interaction as a general means to manipulate electron spins. The spin-orbit interaction allows for electrical control of both single- and two-qubit operations, but unlike the exchange interaction, the spin-orbit interaction generates dressed states of spin and charge where the mixing happens at a high frequency, making the interaction less susceptible to low-frequency noise.

While the general methods we propose in this Letter are applicable in a wide range of situations, we only consider a simplified one-dimensional model where the electrons are localized in quantum dots by an external potential $V(x)$. Physically, we may think of a structure like the one shown schematically in Fig. 1 which was recently realized experi- mentally [8], and we give realistic parameters corresponding to such a system. In our model we include a perpendicular magnetic field $B$ (defining the $z$ direction) and a spin-orbit coupling of the form $p \sigma^{y}$, where $p$ denotes the momentum in the $x$ direction [9]. With two electrons trapped in a double-dot potential the Hamiltonian of the system reads

$$
\begin{aligned}
H & =H_{1}+H_{2}+\frac{e^{2}}{4 \pi \varepsilon_{r} \varepsilon_{0}\left|x_{2}-x_{1}\right|}, \\
H_{i} & =\frac{p_{i}^{2}}{2 m}+V\left(x_{i}\right)+\frac{1}{2} g \mu_{B} B \sigma_{i}^{z}+\alpha p_{i} \sigma_{i}^{y}, \quad i=1,2 .
\end{aligned}
$$

Here $\alpha$ denotes the strength of the spin-orbit coupling, while $m$ is the effective electron mass. Below, we first consider how the spin-orbit interaction allows us to control the spin state of a single electron and then move on to discuss how the combination of the spin-orbit interaction and the Coulomb interaction enables two-qubit operations in a manner analogous to the method used for trapped ions [11].

First, we consider a single electron, and for our analytical calculation we assume that the potential is harmonic, but has a time varying equilibrium position denoted $\bar{x}(t)$,
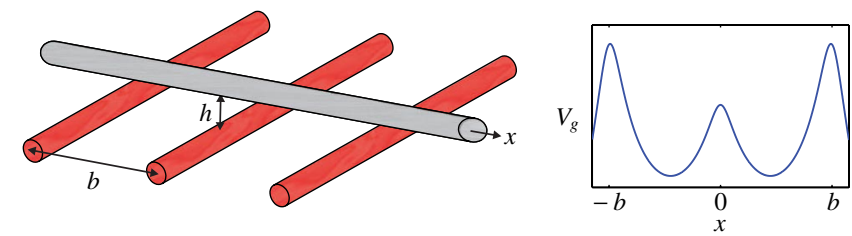

FIG. 1 (color online). A nanowire (light gray) placed above three electrodes. The electrodes are used to define electrostatically a double quantum dot within the nanowire. The electrodes are placed at a distance $b$ apart, while the nanowire is situated at a distance $h$ above the plane of the electrodes. The inset shows a representative curve for the potential $V_{g}(x)$ along the nanowire (the $x$ axis) with the electrodes placed at positions $x=-b, 0, b$, respectively. The shown setup resembles the one used in the experiment in Ref. [8]. 
$V(x)=m \omega_{0}^{2}[x-\bar{x}(t)]^{2} / 2$. We have omitted the subscript $i$, since we are considering a single electron. Physically the time varying equilibrium position can be induced with time varying potentials on the electrodes. We proceed by performing a unitary transformation $H \rightarrow U H U^{\dagger}$ with $U=$ $\exp \left(i \sigma^{y}[x-\bar{x}(0)] / \ell_{\mathrm{so}}\right)$, where we have introduced the spin-orbit length $\ell_{\mathrm{so}}=\hbar / m \alpha$, which characterizes the length scale of the spin-orbit interaction, i.e., in the absence of a magnetic field, a spin along the $x$ or $z$ directions is flipped after traveling a distance $\pi \ell_{\mathrm{so}} / 2$. With this transformation the Hamiltonian becomes

$$
\begin{aligned}
H= & \frac{p^{2}}{2 m}+\frac{1}{2} m \omega_{0}^{2}[x-\bar{x}(t)]^{2}+\frac{1}{2} g \mu_{B} B \\
& \times\left[\cos \left(\frac{2[x-\bar{x}(0)]}{\ell_{\mathrm{so}}}\right) \sigma^{z}-\sin \left(\frac{2[x-\bar{x}(0)]}{\ell_{\mathrm{so}}}\right) \sigma^{x}\right] .
\end{aligned}
$$

We further assume that the renormalized Zeeman splitting $\Delta_{z} \equiv \tilde{g} \mu_{B} B$ ( $\tilde{g}$ defined below) is much smaller than the oscillator energy $\hbar \omega_{0}$, and that the equilibrium position is changed adiabatically with respect to the oscillator frequency $\omega_{0} \gg\left(1 / \ell_{o}\right)[d \bar{x}(t) / d t]$, where $\ell_{o}=\sqrt{\hbar / m \omega_{0}}$ is the characteristic oscillator length. In this limit, we can trace out the motional degrees of freedom and obtain

$$
\begin{aligned}
H_{\text {spin }}= & \frac{1}{2} \tilde{g} \mu_{B} B\left[\cos \left(\frac{2[\bar{x}(t)-\bar{x}(0)]}{\ell_{\mathrm{so}}}\right) \sigma^{z}\right. \\
& \left.-\sin \left(\frac{2[\bar{x}(t)-\bar{x}(0)]}{\ell_{\mathrm{so}}}\right) \sigma^{x}\right],
\end{aligned}
$$

with the renormalized $g$ factor [12] given by

$$
\tilde{g}=g\left\langle e^{2 i(x-\bar{x}(0)) / \ell_{\mathrm{so}}}\right\rangle=g \exp \left[-\left(\ell_{o} / \ell_{\mathrm{so}}\right)^{2}\right] .
$$

The renormalization of the $g$ factor reflects that the qubits states are not pure states of the electron spin, but dressed states of spin and position [13]. Normally, such admixture of spin and position introduces decoherence because the position is coupled to charge fluctuations, but in this case the charge distribution is independent of the spin state, and produces no decoherence in the absence of a magnetic field. With a magnetic field the dressed states are still insensitive to slowly varying electric fields (slow compared to the Zeeman frequency $\Delta_{z} / \hbar$ ), which only shift the equilibrium position, while the spin state follows adiabatically. The dressed states will, however, be sensitive to slowly varying gradients of the electric fields which change the trap frequency $\omega_{0}$. Because of the dependence of $\tilde{g}$ on $\ell_{o}$ in Eq. (4) such fluctuations in $\omega_{0}$ will affect the Zeeman splitting and thereby cause decoherence of the spin states. As we shall see below this is one of the major limitations for the coupling of two qubits, but it will not significantly affect the fast single-qubit operations, provided that the ground state width is reasonably well defined.

Remarkably, the coupling of spin and position can be used to perform single-qubit operations if one applies an electric field with a sufficiently fast variation in time. If we consider the Hamiltonian in Eq. (3) there are two distinct principles for such single-qubit operations. One was considered in Ref. [14], where small amplitude oscillations of the equilibrium position $\left(|\delta \bar{x}(t)| \ll \ell_{\mathrm{so}}\right)$ at the Zeeman frequency produced spin-orbit induced electron spin resonance (ESR) oscillations between the two spin states. The second possibility, similar to Ramsey spectroscopy, for producing spin reversals is to first perform a large rapid change of the equilibrium position for a very short duration. In particular, if we change $\bar{x}(t)$ by $\pi \ell_{\mathrm{so}} / 4$ on a time scale much shorter than $\hbar / \Delta_{z}$, the effective Hamiltonian becomes $H_{\text {spin }}=\Delta_{z} \sigma^{x} / 2$. Consequently, after a time $\hbar \pi / \Delta_{z}$ the spin state has been flipped and we can then change $\bar{x}(t)$ back to the original position. The second procedure has the advantage that it does not rely on any resonance conditions and allows for very rapid manipulation of the spin. Since the time scale can be changed by changing the magnetic field, the spin flip time will in practice only be limited by how fast one can change the voltage on the electrodes. As a particular example of material parameters, we take parameters typical for InAs, i.e., $m=0.027 m_{e}, \hbar \alpha=3 \times 10^{-8} \mathrm{meV} \mathrm{m}$ and $g=14.8$, giving $\ell_{\mathrm{so}}=94 \mathrm{~nm}$. With $B=40 \mathrm{mT}$ and $\hbar \omega_{0}=$ $0.5 \mathrm{meV}$, we get $\ell_{o}=75 \mathrm{~nm}$, so that the Zeeman frequency is $\Delta_{z} / \hbar=(2 \pi) 4.4 \mathrm{GHz}$. The required displacement of the electron spin can be achieved by applying an electric field $E=\pi \ell_{\mathrm{so}} m \omega_{0}^{2} / 4 e=6.6 \mu \mathrm{V} / \mathrm{nm}$, which for a setup like in Fig. 1 with an electrode spacing of $b=$ $500 \mathrm{~nm}$ corresponds to a voltage of roughly $3 \mathrm{mV}$, and the entire spin flip process can be achieved in approximately $0.1 \mathrm{~ns}$.

In addition to being important for performing logical operations in a quantum computer, the ability to perform rapid spin flips also allows the reduction of the leading kinds of decoherence due to the presence of magnetic impurities and the hyperfine coupling to nuclear spins. For electron spins in GaAs a dephasing time on the order of $10 \mathrm{~ns}$ has been reported [4], and we expect a similar time scale for InAs. This dephasing can, however, be reversed by applying pulses, which flip the spin on a much shorter time scale [4].

The spin-orbit interaction can also mediate two-qubit interactions in a very effective way. Returning to the setup shown in Fig. 1 described by the Hamiltonian in Eq. (1), we consider next the possibility of using the dipole moment associated with displacements of the electron charges to couple the two spins. In this one-dimensional geometry the two dots holding each one spin are separated by a distance $d=\bar{x}_{2}-\bar{x}_{1}>0$. We consider the limit where the two electrons are well separated, allowing us to expand the Coulomb interaction term as $1 /\left|x_{2}-x_{1}\right| \simeq 1 / d-\delta / d^{2}+$ $\delta^{2} / d^{3}$, while using $d \gg \delta \equiv\left(x_{2}-\bar{x}_{2}\right)-\left(x_{1}-\bar{x}_{1}\right)$. The first term in this expansion gives a constant contribution to the energy, the second term corresponds to constant forces, which redefine the two equilibrium positions. The last term 
has diagonal terms, $x_{i}^{2}$, which provide a small renormalization of the trapping frequencies. Finally, the interesting term is the cross term $-2\left(x_{1}-\bar{x}_{1}\right)\left(x_{2}-\bar{x}_{2}\right) / d^{3}$, which results in a coupling of the two orbitals degrees of freedom, and hence also, via the spin-orbit interaction, between the two spin degrees of freedom.

To calculate this coupling we go back to Eq. (2) (for each electron) with time independent equilibrium positions $\bar{x}_{i}(t)=\bar{x}_{i}(0)$, and perform perturbation theory in the magnetic field. To second order in $B$ the effective Hamiltonian for a single electron spin is still given by Eq. (3), i.e., $H_{i}=$ $\tilde{g} \mu_{B} B \sigma_{i}^{z} / 2$ (for $i=1,2$ ) plus a spin independent contribution. The cross term that couples the two spins gives rise to an effective spin coupling term given by

$$
H_{\text {spin, } 12}=-\frac{e^{2}}{2 \pi \varepsilon_{0} \varepsilon_{r} d^{3}}\left\langle x_{1}-\bar{x}_{1}\right\rangle\left\langle x_{2}-\bar{x}_{2}\right\rangle,
$$

where the brackets only refer to a trace over the vibrational state, not the spin state. Because the two orbital degrees to this order are decoupled the expectation value separates. To leading order in $B$, the displacement of the electrons can be found from Eq. (2) by first order perturbation theory, and we obtain

$$
\left\langle x_{i}-\bar{x}_{i}\right\rangle=\sigma_{i}^{x} \frac{\tilde{g} \mu_{B} B \ell_{o}^{2}}{\hbar \omega_{0} \ell_{\mathrm{so}}} .
$$

Combining this with the single particle contributions, we arrive at the final effective Hamiltonian for the two spins

$$
H_{\text {spin }}=\tau \sigma_{1}^{x} \sigma_{2}^{x}+\frac{1}{2} \tilde{g} \mu_{B} B\left(\sigma_{1}^{z}+\sigma_{2}^{z}\right),
$$

where the coupling constant $\tau$ is given by

$$
\tau=-\frac{e^{2}}{4 \pi \varepsilon_{0} \varepsilon_{r}} \frac{2 \ell_{o}^{4}\left(\tilde{g} \mu_{B} B\right)^{2}}{\ell_{\mathrm{so}}^{2}\left(\hbar \omega_{0}\right)^{2} d^{3}} .
$$

We stress that this effective Hamiltonian is correct to all orders in the spin-orbit coupling, but only to second order in the $B$ field and first order in the Coulomb interaction between the two electrons. The last approximation can, however, be relaxed without changing the form of the Hamiltonian, but at the expense of a more complicated expression for $\tau$.

To characterize the stability of the proposed coupling mechanism to slowly varying perturbations, such as fluctuations in the gate electrodes, we develop a more realistic model for the double-dot potential $V(x)$. We are having in mind an experimental setup like the one shown in Fig. 1 [8], and, consequently, we consider the electrostatic potential $V_{g}(x)$ created by three parallel electrodes with spacing $b$, each modeled as an infinite line charge, along the $x$ axis running perpendicular to the electrodes at a distance $h$ above the plane of the electrodes. The ratio of the charge density on the left (central) electrode $\lambda_{l(c)}$ and the right electrode $\lambda_{r}$ is denoted $\beta_{l(c)}$, i.e., $\beta_{l(c)} \equiv \lambda_{l(c)} / \lambda_{r}$, which we assume can be controlled via the voltages applied to the electrodes. Moreover, we define $\hbar \omega_{g} \equiv e \lambda_{r} / 4 \pi \varepsilon_{r} \varepsilon_{0}$ and $x_{g} \equiv \sqrt{\hbar / m \omega_{g}}$, in terms of which we express the electrostatic potential as

$$
V_{g}(x)=V_{e}\left(x+b, \beta_{l}\right)+V_{e}\left(x, \beta_{c}\right)+V_{e}(x-b, 1)
$$

with $V_{e}(x, \beta)=-\beta \hbar \omega_{g} \ln \left[\left(x^{2}+h^{2}\right) / x_{g}^{2}\right]$. A representative curve for $V_{g}(x)$ is shown the inset of Fig. 1.

We have implemented numerically on a finite-size realspace grid the two-particle Hamiltonian in Eq. (1) using $V_{g}(x)$ in Eq. (9) as the potential $V(x)$. With $N \sim 100-500$ grid points, the resulting matrix representation of the Hamiltonian is large (dimension $4 N^{2} \times 4 N^{2}$ ), but sparse, allowing for computationally cheap calculations of the low-energy spectrum from which we can extract the coupling of the various spin states. In the left panel of Fig. 2 we show numerical calculations of the coupling $\tau$ as a function of the applied $B$ field. The renormalized Zeeman splitting due to the applied $B$ field is much smaller than the spacing of the orbital levels, and we thus expect Eq. (8) to hold. The numerical results show excellent agreement with the analytic expression. For the parameters used in the figure typical interaction strengths are $\tau / \hbar=(2 \pi) f$, with $f \sim$ $0.1 \mathrm{GHz}$, corresponding to gate times on the order of $1 /(2 f) \sim 5$ ns.

In the right panel of Fig. 2 we show numerical results for the coupling $\tau$ as a function of the voltage applied to the central electrode parametrized by $\beta_{c}$. In order to determine the contribution arising from the bare exchange coupling (due to the Pauli principle and the Coulomb interaction), we also show numerical results for the splitting of the spin states without the spin-orbit coupling. Compared to the bare exchange coupling $J$, which is clearly exponentially dependent on the applied voltage, the spin-orbit induced coupling shows a weaker voltage dependence.
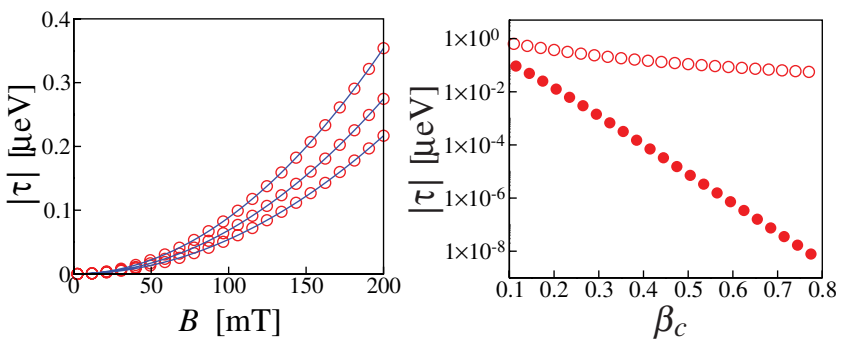

FIG. 2 (color online). Numerical calculation of the coupling $\tau$. Material parameters correspond to InAs and we use $\hbar \omega_{g}=$ $1 \mathrm{meV}, \beta_{l}=1, x_{g}=53 \mathrm{~nm}, h=0.5 x_{g}, b=10 x_{g}$. Left panel: The coupling $\tau$ as function of applied magnetic field $B$ for $\beta_{c}=$ 0.6 (upper circles), $0.7,0.8$ (lower circles). Solid lines show Eq. (8) with the orbital energy spacing $\hbar \omega_{0}$ extracted from the low-energy spectrum and $d$ being the only fitting-parameter. Corresponding to $\beta_{c}=0.6,0.7,0.8$, we have $\hbar \omega_{0}=0.39$, $0.40,0.42 \mathrm{meV}$, and $d=8.3 x_{g}, 8.7 x_{g}, 9.1 x_{g}$, respectively. Right panel: Open circles show the coupling $\tau$ as a function of the applied voltage on the central electrode parametrized by $\beta_{c}$ with $B=40 \mathrm{mT}$. Solid circles show the contribution from the bare exchange coupling $J$. 

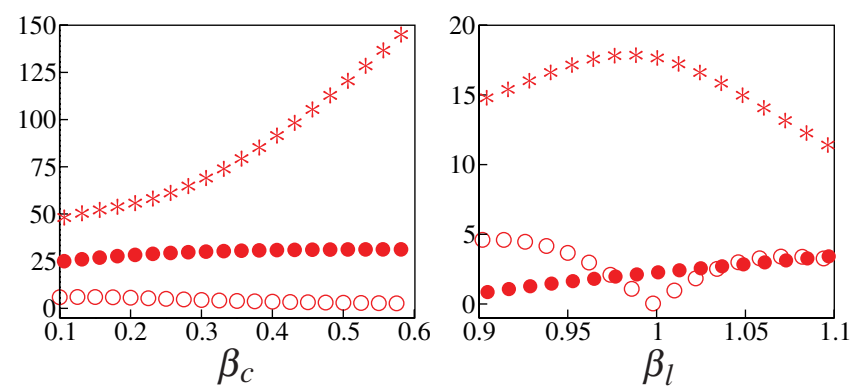

FIG. 3 (color online). Numerical calculations of the sensitivity to fluctuations in the gate electrodes. The sensitivity of the spinorbit induced coupling $\tau$ and the Zeeman splitting $\Delta_{z}$ are quantified by $\left|(1 / \tau)\left(\partial \tau / \partial \beta_{i}\right)\right|, \quad i=c, l$ (open circles) and $\left|(1 / \tau)\left(\partial \Delta_{z} / \partial \beta_{i}\right)\right|, i=c, l$ (stars), respectively. Material parameters correspond to InAs. The other parameters are $B=80 \mathrm{mT}$, $\hbar \omega_{g}=1 \mathrm{meV}, x_{g}=53 \mathrm{~nm}, h=0.5 x_{g}$, and $b=10 x_{g}$. In both panels solid circles show the sensitivity of the bare exchange coupling $\left|(1 / J)\left(\partial J / \partial \beta_{i}\right)\right|, i=c, l$. Left panel: Sensitivity to fluctuations in the central electrode. The electrostatic potential is symmetric, i.e., $\beta_{l}=1$. Right panel: Sensitivity to fluctuations in the left electrode with $\beta_{c}=0.7$.

Fluctuations in the electrostatic environment cause fluctuations of the orbital level splitting $\hbar \omega_{0}$ and the distance $d$. Typically these fluctuations have the form of $1 / f$ noise and concentrating on the dominating low-frequency component, we characterize in the following the sensitivity of the coupling to electrical fluctuations using a purely static calculation by taking derivatives with respect to $\beta_{c}$ and $\beta_{l}$. For the spin-orbit induced coupling $\tau$ given in Eq. (8), we have $\left|(1 / \tau)\left(\partial \tau / \partial \omega_{0}\right) \delta \omega_{0}\right|=4\left|\delta \omega_{0} / \omega_{0}\right|$ and $\mid(1 / \tau) \times$ $(\partial \tau / \partial d) \delta d|=3| \delta d / d \mid$. In order to perform reliable twoqubit operations, both of these quantities must be much smaller than unity, which for the fluctuations imply $\left|\delta \omega_{0} / \omega_{0}\right|,|\delta d / d| \ll 0.1$. In Fig. 3, we show $\mid(1 / \tau) \times$ $\left(\partial \tau / \partial \beta_{i}\right) \mid$ as a function of $\beta_{i}, i=c, l$. The coupling is stable for $\left|(1 / \tau)\left(\partial \tau / \partial \beta_{i}\right) \delta \beta_{i}\right| \ll 1, i=c, l$, implying $\left|\delta \beta_{i}\right|<0.1, i=l, c$ according to the numerical results. This does not impose any unrealistic requirements on the experimental setup. For comparison we also show $\mid(1 / J) \times$ $\left(\partial J / \partial \beta_{i}\right) \mid, i=c, l$ for the exchange interaction $J$, which for fluctuations in $\beta_{c}$ is an order of magnitude more sensitive.

As discussed previously, electrical fluctuations also cause fluctuations of the renormalized Zeeman splitting, $\Delta_{z}$. For $\Delta_{z}$, we have $\left|(1 / \tau)\left(\partial \Delta_{z} / \partial \omega_{0}\right) \delta \omega_{0}\right|=$ $\left(4 \pi \varepsilon_{r} \varepsilon_{0} \hbar m d^{3} \omega_{0}^{3} / e^{2} \tilde{g} \mu_{B} B\right)\left|\delta \omega_{0} / \omega_{0}\right|$. For InAs with $d=$ $500 \mathrm{~nm}, \quad \hbar \omega_{0}=0.3 \mathrm{meV}, \quad B=80 \mathrm{mT}, \quad \mid(1 / \tau) \times$ $\left(\partial \Delta_{z} / \partial \omega_{0}\right) \delta \omega_{0}|\simeq 50| \delta \omega_{0} / \omega_{0} \mid$, implying the stricter condition $\left|\delta \omega_{0} / \omega_{0}\right| \ll 0.01$. In Fig. 3, we show numerical results for $\left|(1 / \tau)\left(\partial \Delta_{z} / \partial \beta_{i}\right)\right|$ as a function of $\beta_{i}, i=c, l$. The results indicate that we must require $\left|\delta \beta_{i}\right|<0.01, i=$ $l, c$ for the renormalized Zeeman splitting to be stable. If this requirement cannot be met in experiments, the prob- lem may be circumvented by encoding a single qubit in a singlet-triplet pair as recently discussed in Refs. $[4,15]$ or alternatively by combining the gate with fast spin-echo pulses implemented by rapidly shifting the position of the electrons.

In conclusion, we have presented a spin-orbit induced mechanism for coherent control of spin qubits in quantum dots. The spin-orbit coupling allows for fast single-qubit operations, and the two-qubit operations are robust against electrical fluctuations in the electrodes defining the double dot. We emphasize that although some of the above conclusions have been made in connection with a specific experimental setup in mind, they also hold at a more general level.

The authors thank G. Burkard, X. Cartoixa, W. A. Coish, A. Fuhrer, A.-P. Jauho, M. D. Lukin, J. Nygård, and J. M. Taylor for valuable discussions and comments. This work was supported by the Danish Natural Science Research Council.

[1] For a recent special issue on solid-state quantum computing, we refer to New J. Phys. 7, 1 (2005).

[2] D. Loss and D. P. DiVincenzo, Phys. Rev. A 57, 120 (1998).

[3] G. Burkard, D. Loss, and D. P. DiVincenzo, Phys. Rev. B 59, 2070 (1999).

[4] J. R. Petta et al., Science 309, 2180 (2005).

[5] X. Hu and S. Das Sarma, Phys. Rev. Lett. 96, 100501 (2006).

[6] A. V. Khaetskii and Yu. V. Nazarov, Phys. Rev. B 61, 12639 (2000); A. V. Khaetskii and Yu. V. Nazarov, Phys. Rev. B 64, 125316 (2001).

[7] D. Stepanenko et al., Phys. Rev. B 68, 115306 (2003); D. Stepanenko and N.E. Bonesteel, Phys. Rev. Lett. 93, 140501 (2004); L.-A. Wu and D. A. Lidar, Phys. Rev. A 66, 062314 (2002); S. Debald and C. Emary, Phys. Rev. Lett. 94, 226803 (2005).

[8] C. Fasth, A. Fuhrer, M. T. Björk, and L. Samuelson, Nano Lett. 5, 1487 (2005).

[9] By a suitable alignment of the magnetic field and definition of the spin operators this form of the coupling applies to any type of spin-orbit interaction linear in $p$ and may have contributions from both bulk and structure inversion asymmetry [10].

[10] L. S. Levitov and E. I. Rashba, Phys. Rev. B 67, 115324 (2003).

[11] J. I. Cirac and P. Zoller, Nature (London) 404, 579 (2000).

[12] S. Debald and B. Kramer, Phys. Rev. B 71, 115322 (2005).

[13] L.-A. Wu and D. A. Lidar, Phys. Rev. Lett. 91, 097904 (2003).

[14] E. I. Rashba and A. L. Efros, Phys. Rev. Lett. 91, 126405 (2003); V. N. Golovach, M. Borhani, and D. Loss, Phys. Rev. B 74, 165319 (2006).

[15] J. M. Taylor et al., Nature Phys. 1, 177 (2005). 